\title{
Information and Mobile Technologies for Promoting Maternal-Child Health Care Status in Rural Areas of Jordan
}

\author{
http://dx.doi.org/10.3991/ijim.v7i3.2540 \\ Edward Jaser \\ Princess Sumaya University for Technology, Amman, Jordan
}

\begin{abstract}
Rural communities in developing countries, due to its geographical and demographic conditions, are faced with many challenges. One of the most pressing challenges which affect the life and welfare of citizens in these communities are health awareness and health services. It is essential to research efficient and cost effective methods to tackle these challenges and be appropriate for developing countries with limited resources. In this paper, we report our findings from implementing a number of ICT interventions to promote maternal-child health care status in rural areas of Jordan. Collected feedback demonstrates the benefits of such system in supporting government activities.
\end{abstract}

Index Terms-Digital Society, Health awareness, ICT for Development, Mobile application.

\section{INTRODUCTION}

Governments in developing countries, and even in developed ones, face continuous challenges with regards to providing good services to its citizens. This is even more challenging when those citizens are living in rural and remote areas. This is due to many reasons, most importantly geographical and demographical conditions and difficulties and inefficiencies with transport and communication infrastructure. Among the most pressing challenges is health services and health awareness provided to these undeserved communities. Around the word, figures and statistics demonstrate that health status in rural communities is inherently lower than those in urban communities. This is easily explainable by the shortages of medical doctors and other health experts in rural communities. Quality health services are usually offered in capital and big cities. This is mainly because those cities offer more opportunities to medical staff to forward their careers in addition to the ease of life and many other advantages. This leaves rural areas and remote communities deprived of specialized and experienced medical staff. It is not difficult to imagine that many medical cases will have to travel to the capital city or other big cities to obtain needed treatment; or wait till the next medical day to happen in their region where a consortia of medical doctors visit rural areas. Shortages of medical expertise can also affect advanced societies. A study by Lenthal et al. [1] demonstrates the challenges facing rural Australia as a result of decreasing numbers of nurses and midwives.

While health services occupy high priorities in any government planning and funding, it has never been sufficient to meet the sheer demand. It is essential to research methods that are effective and efficient to help lessen the huge gap.

Information and Communication Technologies (ICTs) are widely considered by developing countries as the motor of growth, the driver of efficiency and effectiveness and the tool to enhance human development. With the advancement of ICTs and the Internet, communication and web-based technologies can be exploited to address many challenges with relation to improving coverage and obtaining a much needed accurate statistics and information. In recent years there have been concrete evidences on the impact of social networking website in many aspects of life. An obvious and recent example is current events in the Middle East and North Africa dubbed as the Arab Spring. Many claim major roles for social networking tools such as Facebook and Twitter in the dynamics of these events [2]. These tools are changing the way people communicate, receive and exchange information. For example, through ICTs a doctor or medical staff serving rural community can conveniently get updated information about health conditions and illnesses to be used in his day to day practice. Such tools easily attract users as they are discrete, connect large number of individuals and eliminate the middlemen. While most popular networking websites are social in nature, professional networking websites can also be used as an efficient and cost effective tool to tackle issues and problems in society. Appreciating the benefits ICTs bring as an enabling tool, governments around the world are continuously improving ICT infrastructure such as fiber optic networks, internet connectivity and speed and mobile coverage. Recognizing the increased deployment of mobile application to support undeserved communities, Canessa et al. [3] introduced the term Mobile Science (mScience) as a discipline to reflect recent advancement. They explained $\mathrm{m}$-Science its three main activities and its impact on the society: Sensing, Computing and Dissemination of scientific knowledge by the use of mobile devices. They also propose an index to measure the potential and capacity for a community to develop and participate in $\mathrm{m}$-Science activities.

In this paper we report the experience gained from one project concerned with promoting health status of women and children in remote and rural communities of Jordan. This is realized through the design and implementation of a number of ICT interventions appropriate for rural and remote communities to maximize the outreach of information and the utility of the system. The developed 
system serves as: (1) a tool for medical doctors to disseminate awareness information; (2) a source of medical knowledge to rural communities and those clinics serving them; and (3) a tool for clinics to manage immunization of children.

The paper is organized as follow. In Section II we describe some literature work on the impact of ICT in development activities. We present a study about the situation in rural communities of Jordan and the challenges they face in Section III. In Section IV we describe the various stakeholders in our system. We then present, in Section V, ICT interventions proposal to promote maternal-child health status in rural Jordan. In Section VI, we provide an evaluation of our proposed interventions to validate usability and applicability. We conclude the paper in Section VII.

\section{ICT FOR DEVELOPMENT: IMPACT AND OPPORTUNITIES}

With the advancement in ICT technologies and the efforts and fund dedicated to improving infrastructure, many ICT interventions have been introduced to address social challenges including those of rural communities. Cespedes et al. [4] discussed the impact of mobile technology in improving revenue from agricultural activities in Latin America and the viability of this technology in enhancing productivity, facing poverty, and improving social conditions in general. In China, Jun [5] reported evidences showing positive impact of ICT interventions and mobile applications on social challenges such as employment. Cáceres el al. [6] presented an interesting case study about micro- and small enterprises usually operating in poor neighborhoods in Lima, Peru. The study demonstrated the added value of ICT and mobile applications in increasing productivity and improving marketing activities and client relations.

ICT have also been used to empower women in those areas where they face challenges in their everyday activities. This has been evident in the work of Sharma et al. [7]. They studied the impact of ICT initiatives on women in the districts of Kanpur and Lucknow in India. They reported increase of income, change of attitude and improved ICT skills of women participating in the study.

Smith et al. [8] reported on the important roles that mobile technologies as a platform for multidimensional enhancement of capabilities in developing countries. They reflected on policy issues and implications of mobile communication in increasing noneconomic capabilities and freedoms.

In Nicaragua, a research project was initiated targeting rural communities in extreme poverty [9]. The project aimed at strengthen medical follow-up on the health and nutrition status of mother and children in those communities. One attractive characteristic of the developed system was its low cost which can help in replicating it to other regions. Another interesting work is the initiative by Raja et al. [10]. They proposed collaborative model of a health information system for rural communities and the challenges and opportunities of this global issue. They validated the effectiveness of a collaborative model that joins technology partners, teaching institutions, and healthcare providers. Medical awareness using technology was also approached by Maeda et al. [11]. They proposed an application framework based on MVC, for health awareness and education using interfaces such as web and e-mails.

All aforementioned initiative have reported behavioral impact on the served communities and provided proof that ICT can actually benefit and support undeserved communities. As a result it is not surprising governments and not for profit organization are focusing on such initiative to enhance their services and extend its outreach. In this paper we report on our experience from an ICT initiative specific to the need and requirements of Jordan unprivileged communities.

\section{RURAL COMMUNITIES IN JORDAN: FACTS AND CHALLENGES}

Our project has been targeting rural communities of Jordan. We have been engaged in a phase of data collection to discover facts and conditions to help us when designing the ICT interventions. In this section, we report some of these facts and detail some of the main challenges facing those communities.

\section{A. Jordan Facts}

Jordan is a small country with an area of approximately $92,300 \mathrm{~km}^{2}$ and an estimated population of $6181 \mathrm{~K}$ people. There are 12 Governorates in Jordan (Figure 1). According to the Statistical Yearbook of Jordan (published in 2007 by Department of Statistics [13]), the population is divided between urban communities (those localities of $5 \mathrm{~K}+$ inhabitants) and rural communities. Almost $18 \%$ of the population is classified as rural and $82 \%$ as urban.

Amman, Irbid and Zarqa are the biggest cities of Jordan and more than $62 \%$ live in these governorates.

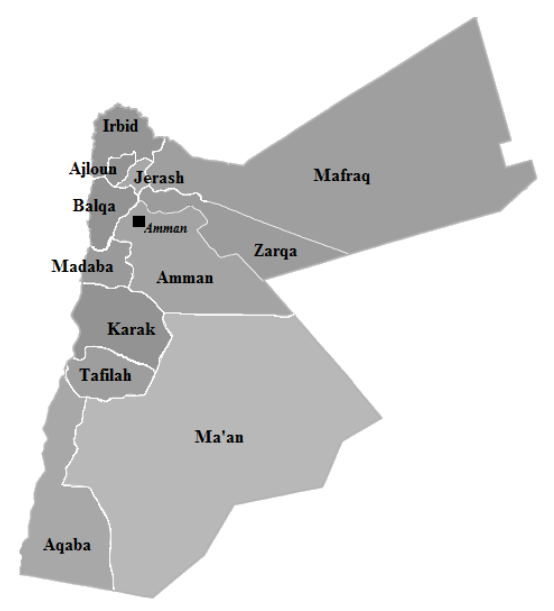

Figure 1. Map of Jordan showing its 12 governorates.

The remaining governorates are larger in area and have higher percentage of rural communities. Large percentage of population in these governorates live in scattered communities in the form of small villages and towns and have a significant number of moving population (or Bedouins). Figure 2 shows the percentage of rural population out of the total population in each governorate. Karak, Mafraq and Ma'an are the governorates with the most rural and remote communities. For example, $64 \%$ of the total population of the governorate of Karak is 
classified as being rural population. Ma'an has a percentage of almost $45 \%$ classified as rural communities. The challenge in this governorate, the largest in Jordan, is that its rural population is scattered throughout a large area. Amman, Zarqa, Irbid and Aqaba have the lowest percentage of rural communities. In general the governorates in the south tend to have the most rural and remote communities and also the most challenging condition in terms of transportation, communication, outreach and other infrastructure issues. We opted to focus our activities and our pilot project in selected areas of southern Jordan.

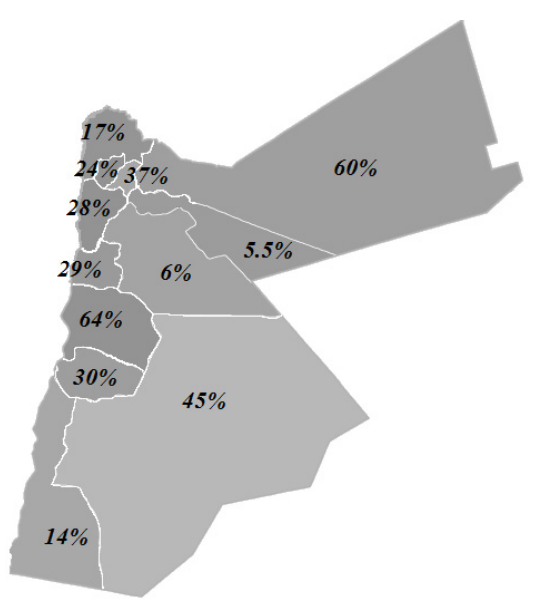

Figure 2. Map showing percentage of rural communities in each governorate.

\section{B. Health Facts and Challenges}

Birth rate are in Jordan are average compared to the world [12]. Crude child rate (per 1000) is estimated around 28 and population growth rate is $2.3 \%$. Death rate among children per 1000 is around 7. In general, health services in Jordan are considered advance compared to the region. However, most of the advanced and well equipped hospitals and clinics are privately owned and are concentrated in big cities. This has affected the health status in rural communities. For example (1) rural children are more likely to be chronically malnourished than are urban children (12 percent versus 7 percent); (2) The prevalence of anemia among children is higher in rural areas than in urban areas; and Perinatal mortality is higher among women in rural areas. We can find much similar statistical evidences in $[12,13]$ to support this premises.

Jordan Ministry of Health is striving to continuously enhance and widen services to rural and remote communities. Governorates are served with different types of medical centers such as Mother and Child health centers, village centers and health centers. However, there will always be efficiency and effectiveness issues. One problem noticed during the research team visits to some health centers serving the south of Jordan was the effectiveness of health leaflets and brochures and other awareness means to residents of these communities. A better way of managing health information and awareness is of importance to these clinics.

\section{Partnering with public institutions and national initiatives}

One important success factor when engaging in development project is to identify those stakeholders that are serving local communities through the various national initiatives. We identified two main stakeholders: Knowledge Station and Jordan Ministry of Health $(\mathrm{MoH})$.

Knowledge station is an initiative designed to give citizens of Jordan the opportunity to experience the benefits of living in an IT-connected world specially those living in rural or remote communities. It is estimated that by August 2012, more than 1.76 million citizens had benefited from services provided by those stations. The stations are well distributed across all governorates. The Objectives of the Knowledge Stations include:

- Bridging the digital divide between the governorates and different regions in the Kingdom;

- Introducing ICT to the different localities in Jordan and encouraging the use of ICT in the daily lives of citizens;

- Alleviating IT illiteracy by providing training in ICT;

- Encouraging the use of the National Information System for retrieving local information;

- Enhancing the use of the Internet for socio-economic development at the community level;

- Enhancing local community skills through ICT training;

- Enhancing competition among citizens by increasing their knowledge in ICT.

- Preparing the local communities to get involved in the egovernment project.

The Knowledge Station proved to be a logical partner to our project. The ICT infrastructure they have are distributed across rural communities and can be effectively used to make people aware of the project and train them to use the system. Also it played an important role in data collection, gather feedback, and evaluation.

We also relied on the information and services provided by Jordan Ministry of Health to support our project activities. Particularly, the ministry has helped in identifying the medical centers that are facing challenging conditions.

\section{Data Collection}

To get better understanding of the situation in rural areas, we designed a set of questionnaires and structured interviews. The aim was not to obtain data to release statistics and figures but rather to get familiar with the situation in those communities to better design appropriate ICT interventions. We distributed around 200 questionnaires and conducted around 50 interviews.

Questionnaires were distributed to members of rural communities randomly selected with the aid of the Knowledge Stations. 191 questionnaires were collected back. Following are the analysis of various parts of questionnaire.

It was noticed that the Knowledge Stations are a popular choice to obtain internet connectivity in rural and remote communities with active participation in training activities and other events organized by the stations. The main usage of Internet were viewing news websites, using search engines to search for different topics and accessing e-mails. Majority of questioned users spend only from 1 to 
2 hours daily surfing the web (Figure 3). This was attributed to lack of suitable content for them to consume.

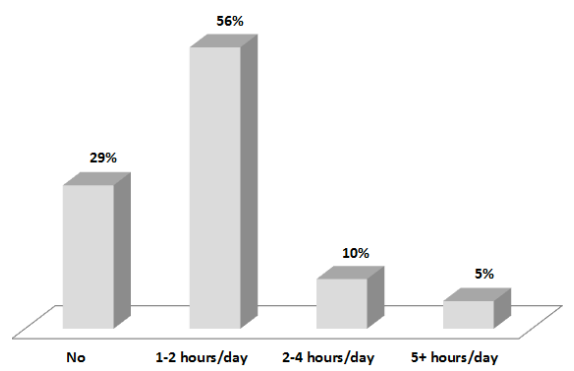

Figure 3. Internet penetration in terms of number of hours spent.

Health topics were among searches initiated by users and it was a popular options. Figure 4 shows that mainly, out of medical topics, users were interested in dietary information (around 26\%), various diseases and cures $(18 \%)$ and topics on alternative medicines (15\%). Users interviewed were not impressed with the availability (in terms of quantity and quality) of medical articles in Arabic.

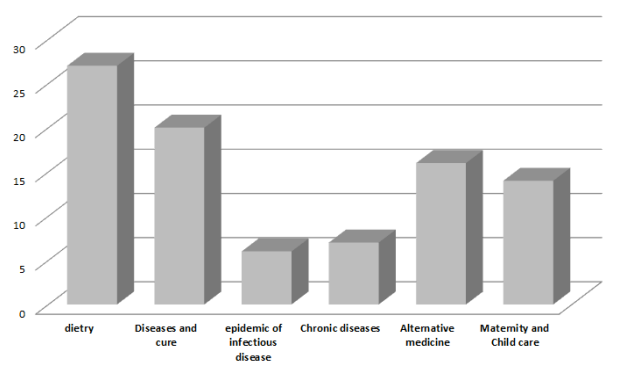

Figure 4. Interesting health topics most searched by stakeholders.

It was interesting to notice that majority of questioned users think that medical doctors are the most appropriate resource for medical information if they are available (Figure 5). The issue of the difficulty of having access to a medical doctor was raised by significant number of users. Other users would rely on Internet, TV and other media or seeking advice from family member to get required health information.

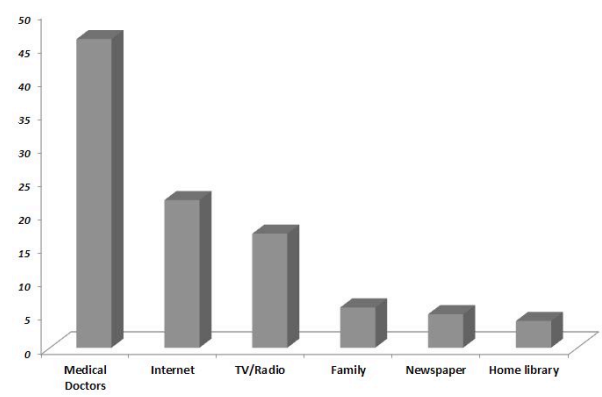

Figure 5. Resources for health information.

Regarding mobile penetration, it was noticed that the majority of the people who answered the questionnaire had at least one mobile phone (see Figure 6). Most of these phones are basic and cheap units rather than smart phones.

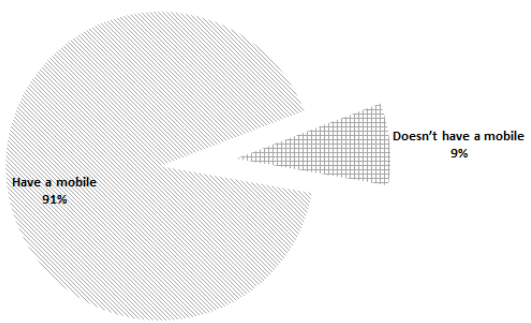

Figure 6. Mobile penetration in terms of owning a mobile line.

As for the usage of the mobile phone, mainly it is used to make/receive phone calls (more than $70 \%$ as first choice) and send/receive SMS messages (Figure 7). Small number of users reported that they use their mobile handset to access the internet.

One of the things we investigated was the usage of the online and mobile e-Government services provided by Jordan Ministry of ICT. Most questioned and interviewed users were not aware what services were provided online. Some reported on the difficulty of interacting with these services.

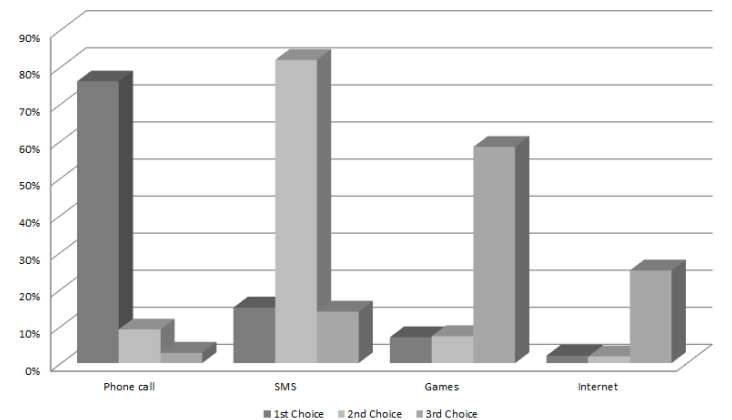

Figure 7. Usage of Mobile Phones in rural areas. Engaging in phone calls and sending/receiving messages are the most functionality used.

Another initiative that we partnered with was the national SMS gateway through which the e-Government was providing services to enable citizens to access important information pertaining to transactions and governmental procedures easily anywhere and at any time using push and pull SMS messages. Users were aware of the availability of this service but almost $32 \%$ of users actually used it (see Figure 8).

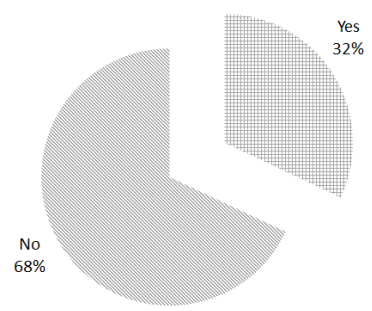

Figure 8 . Popularity of the e-Government services. $32 \%$ of questioned users use various online services provided by the government.

As mentioned earlier, the conducted data collection was aimed to better understand the behavior of users in rural 
and remote communities rather than obtain publishable statistical figures. Interesting facts learned from this phase that while residents of these communities have limited access to Internet there are initiative (such as KS) that are attempting to bridge the connectivity gap. One problem though facing these initiatives is availability of suitable and relevant content. There is a need to enrich content targeting this slice of users. Another interesting fact is the excellent penetration of mobile phone. This qualifies it to be the most appropriate communication channel to deliver information and collect feedback and questions. We also learnt that medical information is important and there is serious shortage of health awareness content. Our focus was put on improving awareness and services offered to mothers and children given the importance of this topic both at government level and among communities.

\section{STAKEHOLDERS AND THEIR REQUIREMENTS}

One of the early stages in the project was to identify the primary stakeholders who directly interact with the system [14]. We have categorized the people who work with the system into four groups:

- Medical content consumers: individuals who use the system to obtain medical knowledge through the various channels available for the project. They can either be registered users or use the system as anonymous.

- Medical content producers: those who populate the database with required medical knowledge whether diagnosis, awareness ... etc. To be able to use the system, they need to be registered.

- Medical experts: those who filter incoming information to ensure validity and consistency. They need to be registered and be granted privileges to approve any piece of incoming/outgoing information.

- Administrators: those who will manage system users and manage accounts.

There are also secondary stakeholders who affect and are affected by the system: local clinics and mobile clinics, NGOs working in rural areas and e-Government portal.

There are three main type of users, who can carry out six different types of interactions with the system: (1) guest users can browse the public information on the project and view the various content provided by the website, as well as ask anonymous questions to the medical experts; (2) registered users, in addition to having access to the same functionality as guests they can participate in the activities where only registered users are allowed to participate such as forum, adding content (for medical experts) and supervising content to check validity and correctness (for content supervisors); (3) administrator who additionally take the responsibility of user and system management activities.

The system was developed to meet the following main requirements: (i) allowing content consumer to interact with the system and access stored knowledge and provide them with the tool to post medical questions; (ii) the system able to store information about stakeholders who would like to be contacted to receive awareness information and information about organized events; (iii) allowing content producers (medical expert) to log in to the system and maintain medical knowledge and answer questions; (iv) allowing content supervisor to $\log$ in to the system and receive notification of new material added to the system and to rate them and filter them accordingly; (v) allowing system administrator to define new users and manage existing ones as well as manage system components; and (vi) allowing clinics serving rural and remote areas to manage vaccination process of children.

When developing health related system for use in rural areas, issues related to interacting with potential users that promote willingness to engage in this system would play a critical role in developing trustworthiness [15]. This has influence our choice of user interface and motivated us to work closely with identified stakeholders. The system has been designed to be replicable and scalable.

\section{ICT INTERVENTION TO PROMOTE MATERnAL- Child Health Care Status}

In this section we provide details on the ICT interventions design to promote health status of mother and children in rural area. We identified three main component of the system:

- Component 1: the dissemination and delivering of awareness information in efficient, convenient and cost effective way;

- Component 2: enable users to post medical questions and interact with medical staff using digital means; and

- Component 3: manage automatically the vaccination process to improve vaccination coverage among rural and remote communities.

\section{A. Architecture Design}

The design of the system is driven by the requirements of the identified stakeholders. The design (illustrated in Figure 9) is based on database driven web and mobile applications.

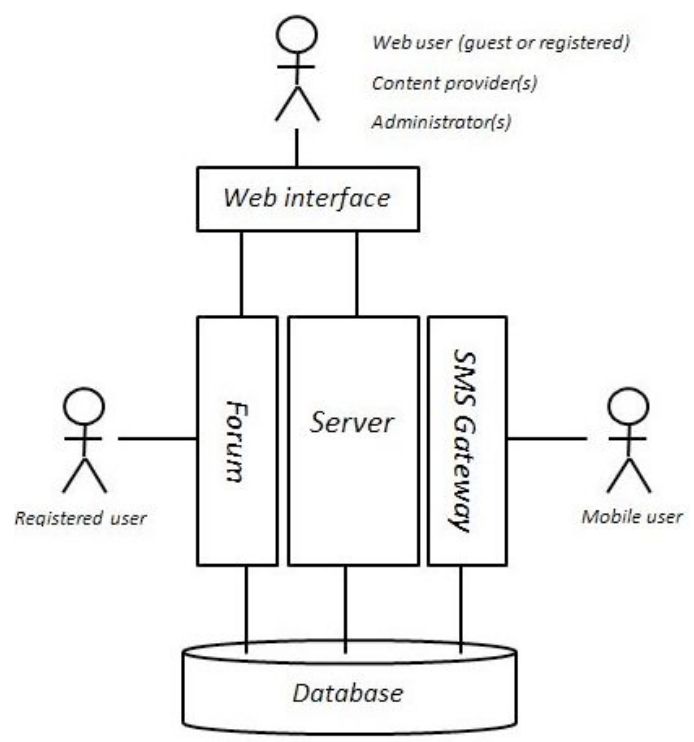

Figure 9. Architectural design of the proposed ICT interventions to promote maternal-child health status.

The system has three interface channels: a website containing awareness content and a facility to enable question/answer interaction between medical staff and users, a mobile interface to disseminate and deliver awareness SMS and vaccination information and 
reminders and a forum to enable discussion between users. The system allows various types of access: registered users, administrators, mobile users as well as anonymous users. Of course anonymous users can view information and are not allowed to enter or modify data.

\section{B. Component 1: Health Information Awareness}

This component is concerned with enabling medical staff and Ministry of Health employees to post awareness information about maternity and children health. The information can be textual, images, audio and video content. The medical staff needs to be registered in the system to add content. Once a new item has been added it awaits the approval for content supervisor to validate it. Once the content is validated the users (whether registered or guest) can view it. Example contents include awareness posters (such as smoking related or cancer information), medical articles, awareness videos (as the ones aired on TV) as well as audio advices.

\section{Component 2: Questions and Answers}

The component enables users to seek medical advice digitally. The idea behind this service is to give the opportunity for those who have limited access to medical doctors to establish a communication channel with medical doctors in big cities (e.g. Amman). The system will enable registered user, through web interface, to post a question. The system then notifies registered medical experts that a new question has been added to the database. The expert can login to the system and post an answer. More than one answer can be posted by different experts. The answers will not be visible on the website till a content supervisor approves it.

\section{Component 3: Vaccination Management System}

Statistics [13] shows the vaccination rate among communities in rural areas (such as in the governorates of Ma'an, Tafiela and Aqaba south of Jordan) are lower than other governorates. The reason can be attributed to lack of effective awareness, illiteracy rate, lack of reminders and lack of medical personnel. Any intervention should address these issues.

The high-level requirements of this component are to:

1) Register new born babies with the system and calculate the vaccines based on the vaccination schedule maintained by the Ministry of Health.

2) Issue reminders to parents reminding them on the date and the type of the due vaccine.

3 ) Issue reminders and volume information to clinics on the number and types of vaccination they will be expecting to perform in a specific day to make sure they secure the needed quantities.

4) Provide awareness information to parents to help them establish the importance of the vaccines for their children.

Given the identified requirements, we designed the vaccination management system based on a clear identified scenario and simple workflow [16].

Workflow: Clinic staff registers new born babies with the system. This can be done either using the internet (website) or, if the infrastructure is not there, using smart phone over 3G networks. The application can be downloaded and used with any java enabled phone. The clinic personnel need to capture basic information about the child (name, date of birth, weight, height and contact details of parents) and send the information to the system as an SMS message. Once the information of a new child have been received and stored, the system uses the vaccination schedule issued by the Ministry of health to calculate the dates of the vaccines for the registered child and store them on the database. The system continuously checks the database to produce a report of which children due to be vaccinated in a certain day. The system automatically sends to the parents a reminder on the next vaccine for their child and at which clinic. Also, the system sends statistical and volume information to clinics about expected children and vaccines at a certain date. When the vaccination of a child takes place, the clinic personnel send this information to the system to maintain the child record.

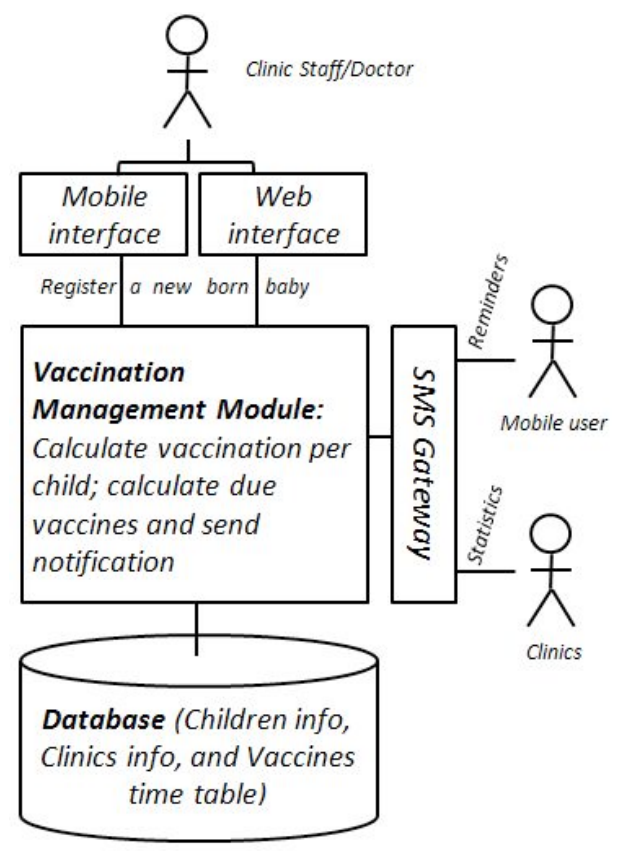

Figure 10. Main components of the Vaccination Management System.

The database maintains up-to-date record of vaccination to be used by decision makers at the ministry to obtain information for the purpose of reporting and planning. Figure 10 depicts the architectural design of the system, the input/output channels and support information.

One improvement added later to the system was to enable voice reminders to be sent for citizens who were not able to read messages.

\section{Evaluation}

\section{A. Pilot System Deployment}

It was extremely difficult to evaluate the system nationwide due to financial requirement and project's limited budget. Therefore we opted for selecting three medical centers serving poor and remote communities in the south of Jordan. The selected clinics were selected with the help of Jordan Ministry of Health. 


\section{B. User Feedback}

We report in this section the feedback we obtained from both medical staff serving in local clinics as well as patients. We opted for questionnaire and interviews as a tool to gather feedback. Thirty KS staff and medical staff (both doctors and nurses) and twenty patients were interviewed. We also collected forty three evaluation questionnaires from residents in those communities where clinics were chosen for the pilot phase.

All KS staff agreed the importance of our system as a mean to increase specific content targeting residents of rural and remote communities especially with regards to component 1 and 2. This will support the mission of the knowledge station in serving those communities.

Feedback from medical staff showed interest in Component 1 . They regarded this module and information delivery to mobile phones as an important tool to reach a wider number of citizens. Majority agreed that this will be a more attractive tool to disseminate information compared to conventional methods (e.g. leaflets). They also considered component 3 as a very important tool to local clinics for the smooth management of vaccination. Component 2 did not receive much appreciation. This was because medical experts did not want to have the responsibility of misdiagnosis. Most of interviewed experts agreed that it was far too early to discuss remote diagnosis in Jordan and they demand that a further research on a larger scale on this topic should be performed. They also pointed on the importance of reviewing the regulation and legal framework for remote diagnosis.

Feedback from local clinics viewed the vaccination system as the most appropriate replacement for the paper recording system they have using and the conventional methods of issuing reminders or calculating daily needs of various vaccines.

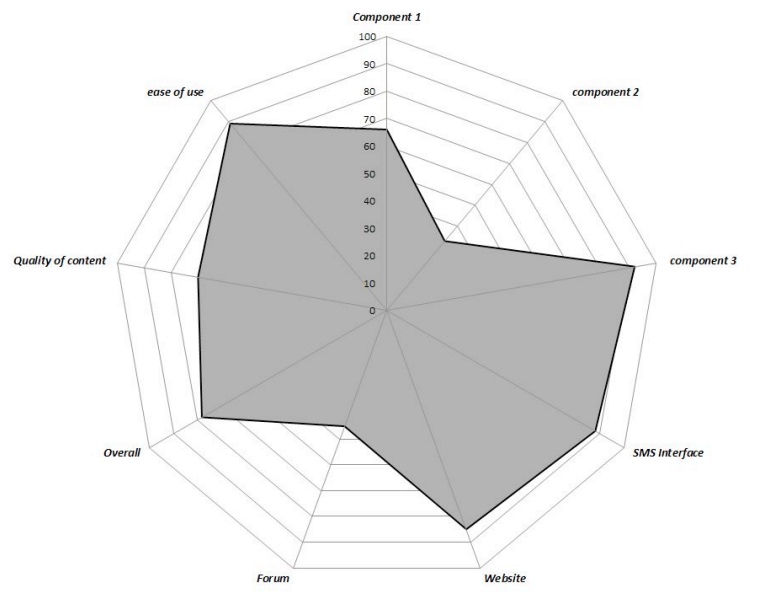

Figure 11. Feedback analysis. Positive answered is shown.

Overall feedback from citizens were positive and in favor of using the system. When asked about the overall idea of the system, more than $78 \%$ considered that the system is a necessity and will certainly help their community. Automatic vaccination management was viewed as very important tool by citizens given that most questioned citizens reported on issues with remembering vaccination date and type for their children or with the smooth conduct of vaccination at clinics. Significant number of citizens questioned the benefits from having a questions/answers facility or having yet another forum to discuss medical topic and whether they can rely on them. Good feedback obtained regarding the web and mobile interfaced and their ease of use. Quality of content was significantly criticized. This is explained by the fact that only small numbers of textual, audio and visual content were collected form previously generated content and there were limited resources to invest in developing detailed content.

Overall, it is evident from the positive feedback (see Figure 11) the benefits from implementing such intervention using ICT technologies. Quantitative evaluation and longitudinal studies is required over a long period of time to record impact of the system on health status in underserved communities. It is important to cover more clinics and more regions across Jordan.

\section{CONCLUSION AND FUTURE WORK}

In this paper we described an ICT interventions aimed at enhancing the health status of mother and children in rural and remote communities of Jordan. The purpose of the system was to provide a proof of concept on using technology to enhance services to undeserved communities. The system deployment was limited to three local clinics in the south of Jordan. Feedback on this pilot implementation demonstrated promising impact. One obvious and important future work is to scale the system to include more clinics to obtain quantitative results to perform solid impact analysis.

\section{REFERENCES}

[1] S. Lenthall, J. Wakerman, T. Opie, S. Dunn, M. MacLeod, M. Dollard, G. Rickard, and Sabina Knight, "Nursing workforce in very remote Australia: characteristics and key issues," Australian Journal of Rural Health, 19, pp. 32-37, 2011. http://dx.doi.org/10.1111/j.1440-1584.2010.01174.x

[2] M. Papic and S. Noonan, "Social Media as a Tool for Protest," web article http://bit.ly/fE1Qnt, Stratfor Global Intelligence. February 3, 2011. accessed 15/02/2012.

[3] E. Canessa, M. Zennaro, "A Mobile Science Index for Development," International Journal of Interactive Mobile Technologies (iJIM), volume 6, No 1, pp. 4-6, 2012.

[4] L. Cespedes and F. Martin, "Mobile Telephony in Rural Areas: The Latin American perspective," The i4d print magazine, VII No. 9 pp. 10-11, January-March 2011.

[5] L. Jun, "Mobile Social Network in a Cultural Context," In E. Canessa and M. Zennaro: m-Science: Sensing, Computing and Dissemination., ISBN:92-95003-43-8. pp. 211-240. 2010.

[6] R. Cáceres, A. Agüero, M. Cavero and C. Huaroto, "The Impacts of the Use of Mobile Telephone Technology on the Productivity of Micro- and Small Enterprises: An Exploratory Study into the Carpentry and Cabinet-Making Sector in Villa El Salvador," Journal of Information Technologies \& International Development, volume 8, issue 4, pp. 77-94, 2012.

[7] C. Sharma, S. Sharma and U. Subhedar, "Putting ICTs In the Hands of the Women of Kanpur and the Chikan Embroidery Workers of Lucknow," Journal of Information Technologies \& International Development, volume 4, issue 2, pp. 11-16, 2007.

[8] M. Smith, R. Spence and A. Rashid, "Mobile Phones and Expanding Human Capabilities," Journal of Information Technologies \& International Development, volume 7, issue 3, pp. 77-88, 2011.

[9] S. Bravo, M. Valero, I. Pau, J. Duarte, M. Carrillo and J. Blandino, "A Tele-Health Communication System for Underserved Children in Rural Areas of Nicaragua," Journal of Information 
Technologies \& International Development, volume 8, issue 4, pp. 205-221, 2012.

[10] U. Raja, D. J. McManus, J.M. Hardin and B. C. Haynes, "Collaborative Rural Healthcare Network: A Conceptual Model," International Journal of Interactive Mobile Technologies (iJIM), volume 5, No 3, pp. 20-23, 2011.

[11] T. Maeda, T. Okamoto, Y. Fukushige, and T. Asada, "Mobile Application Framework for Health Care Education," 7th IEEE Consumer Communications and Networking Conference, pp. 1-2. January 2010.

[12] Department of Statistics, "Jordan 2009 Population and Family Health Survey: Key Findings,” Amman, Jordan 2009.

[13] Department of Statistics, "Jordan 2007 Population and Family Health Survey: Key Findings,” Amman, Jordan 2007.

[14] E. Jaser and I. Ahmad, "ICT Intervention to Enhance Health Services to Mothers and Children in Remote Communities in Jordan," MobiCASE, volume 95 of Lecture Notes of the Institute for Computer Sciences, Social Informatics and Telecommunications Engineering, pp. 303-310. Springer, 2011.
[15] A. Furling, S. Lee, "The influence of user-interface usability on rural consumers"e trust of e-health services." International Journal of Electronic Healthcare, volume. 2 issue 4, 2006.

[16] E. Jaser and I. Ahmad, "Towards the Automatic Management of Vaccination Process in Jordan," Proceeding of the Sixth International Conference on Digital Society ICDS, pp. 50-54, Valencia, Spain 2012.

\section{AUTHORS}

E. Jaser is assistant professor at the King Hussein School for Computing Sciences at Princess Sumaya University for Technology and was the lead ICT researcher at the Royal Scientific Society till 2010 (e-mail: ejaser@psut.edu.jo).

This work was funded and supported by the International Development Research Center (IDRC), Canada and the Royal Scientific Society, Jordan. Submitted 28 February 2013. Published as re-submitted by the author 26 June 2013. 